\title{
Implementation of MPLS Layer 3 VPN in IPV6 (MPLS 6VPE) Networks
}

\author{
Gayathri.G ${ }^{1}$, Kaviya.K ${ }^{2}$ \\ Assistant Professor, Department of Computer Science, \\ Ponnaiyah Ramajayam Institute of Science and Technology PRIST University, Thanjavur ${ }^{1}$ \\ M.C.A., Scholar Department of Computer Science, \\ Ponnaiyah Ramajayam Institute of Science and Technology PRIST University, Thanjavur ${ }^{2}$
}

\begin{abstract}
Today several organizations are in a process to adopt IPv6 addressing scheme. Since IPv6 is not backward compatible with IPv4, both IPv4 and IPv6 are going to work in parallel till IPv6 is fully deployed .Security in IPv6 is mandatory, but in IPv4 security scheme is optional. IPv6 packets are travelling through IPv4 network in transition mechanism. The IPv6 packet faces several security threats while it travels through IPv4 network. The transition remains an issue today for many enterprises which is a tedious and error prone task for network administrators. Routing protocols route the packet with their own metrics. The network administrator could not implement his own routing methodology in a routing process.In this project we are taking IPv6 network in GNS3 simulation tool and implement the IPSec scheme. Unauthorized routers are introduced in our network and we have to ensure that services for them are denied by our network routers. In the same network we are implementing the Policy Based Routing (PBR). Routers normally forward packet to destination addresses based on information in their routing tables. By using policy based routing we can implement policies that selectively cause packets to take different paths based on source address, protocol types or application types. Therefore PBR overrides the routers normal routing procedures. By implementing both the schemes in our IPv6 network, the IP packets are delivered safely and also take the path as desired by network administrator.
\end{abstract}

Keywords: Policy Based Routing (PBR), IPv4 network, IPv6 network, Quality of Service (QoS)

\section{INTRODUCTION}

Computer evolution from IPv4 to IPv6 is the biggest transformation in Internet infrastructure since its beginning. The process is (and will be for many years) very complex and resources (human, money)consuming. It must be expected that the transformation will have huge impact on many aspects of Internet services: network performance, data security, economy. In general, security issues in IPv6 are not better or worse than in IPv4, they are just different. There are risks related to all security features: confidentiality, integrity and availability. For many years we will live in a dual IPv4/IPv6 environment. The security issues could become complex to deal with in terms of implementation and configuration. In dual-stack architecture used in transition phase the problems resulting from IPv6 introduction may have unforeseen effects on IPv4 processing, affecting not only new services but also old services (based on IPv4). The IP transition phase is an important research area of many teams (e.g., 6net [1], IPv6fix [2] in Japan, USGv6 [3] in the USA). There are some resources on different aspects of IPv6 security. Complete list of new threats and risks related to IPv6 is very long and it is very probable that we do not know all threats and risks. In the paper we try to present comprehensive survey on IP security issues with emphasis on the security in IPv4 toIPv6 transition phase. Section II presents some remarks on solutions to the transition period problems. Main part of the paper (Section III) is dedicated to several issues related to security of IPv6 deployment and transition phase. General conclusions are given in the last part of the paper.

\section{LITERATURE REVIEW}

A survey of ipv6 protection method and their utilization in combination with online routing method is presented in this session. usually fault management methods are pre-establish backup path to recover ipv4 after a concept of ipv6.we define new Quality of Service (QoS) routing schemes with protection in IP multi protocol label switching over optical network the novelty of the proposed routing schemes of ipv6.IPV6 could combine mobile network and fixed wireless network closely which brings great convenience to peoples live mobile ipv6 is described the current main switch method are summarized and the typical methods are detailed and compared in this paper and at last the future research hotspots are proposed in ipv6 


\section{EXISTING SYSTEM}

The availability of an almost unlimited number of IP addresses is the most compelling benefit of implementing IPv6 networks to IPv4, IPv6 increases the number of address bits by a factor of 4, from 32 bits to 128 bits. The 128 bits provide 4.3 billion addressable nodes, which can satisfy any predictable address space requirement. Theoretically speaking, IPv4 can provide at most 4.3 billion addresses whereas IPv6 can provide at most 4.3 billion $\times 4.3$ billion $\times 4.3$ billion $\times 4.3$ billion An IPv6 address consists of 128 bits, which can provide an address space and network prefix far greater than that of IPv4. Therefore, a network can be hierarchically deployed with IPv6. The same organization can use only one prefix in the network. For ISPs, a greater address space can be obtained. Therefore, ISPs can every one buyer into one prefix and distribute the prefix. With hierarchical convergence, the global routing table contains few address entries and thus the forwarding efficiency is higher

\section{Disadvantages}

- $\quad$ Routing table entries for such along and many networks causes load on the network infrastructure

- It very difficult to remember an addresss88 23:A123:0000:0000

- $\quad$ Dual stack approach must be configured manually if old devices don't support ipv6

- $\quad$ No longer supported for older devices.ie(IPV4)

\section{PROPOSED SYSTEM}

Improved version of IPv4.IPv6 is a packet-switched internet working. Pv6 provides end - to - end datagram transmission. IPv6 is coupled from any particular link layer as it uses IPv6neighbor discovery instead of ARP Improves the robustness of the protocol Provides solid security for internet communication

\section{Advantages:}

- $\quad$ Provides more address space

- $\quad$ More powerful internet(128bit versus ipv4's current 32bit)

- $\quad$ Offers and overall larger scale internet-which again will be needed in the furture

- $\quad$ Address allocation is done by the device itself

- $\quad$ Support for security using(IPSEC)internet protocol security

\section{MPLS (MULTI PROTOCOL LABEL SWITCHING)}

Switching is the process by which, two circuits are interconnected for exchanging information. Information is in the form of either analog or digital. In electro mechanical era, information was in the form of analog. Presently, on formation is in the form of digital. In order to interconnect the circuits, supporting the digitized information, suitable digital switches are designed.

Digital Switches are classified as

(1)Circuit switch

(2) Packet switch

Apart from the above models of switching, Multi-Protocol Label Switching model is configured in Packet Switch Area.

Circuit Switches: Route switch mainly supports the switching the voice paths. Digital spectrum is divided into equal parts $(64 \mathrm{kbps})$. Circuit switch uses these $64 \mathrm{kbps}$ path for voice switching. Voice samples of a particular conversation should reach the destination sequentially through the $64 \mathrm{kbps}$ digital path by maintaining maximum permissible delay of 125 us, to avoid the loss of intelligence. In order to satisfy the above conditions, switched path should be permanent until the end of the conversation. . Hence, the routing becomes connection oriented. No other user also can intrude in that path. Also the route paths can be categorized according to the type of services and class of services.

\section{Class of Services \\ - $\quad$ Emergency Services Routes \\ - $\quad$ Special Services Routes}

Packet Switches: Instead of dividing the digital spectrum, entire message is divided into packets, addressed and numbered. Packet switch sends the addressed and numbered packets one by one to the destination, in different routes, by using the entire spectrum available in last week. For an example, if the packet size is $2 \mathrm{mb}$ then the packet switch uses the 2 mbps digital spectrum for the period of one second. At destination, packets are arriving randomly at different 
time. Even the first packet may arrive lastly. Receiver has to wait until all the packets are received. Then packets are arranged sequentially and then converted as message.

\section{MPLS Architecture}

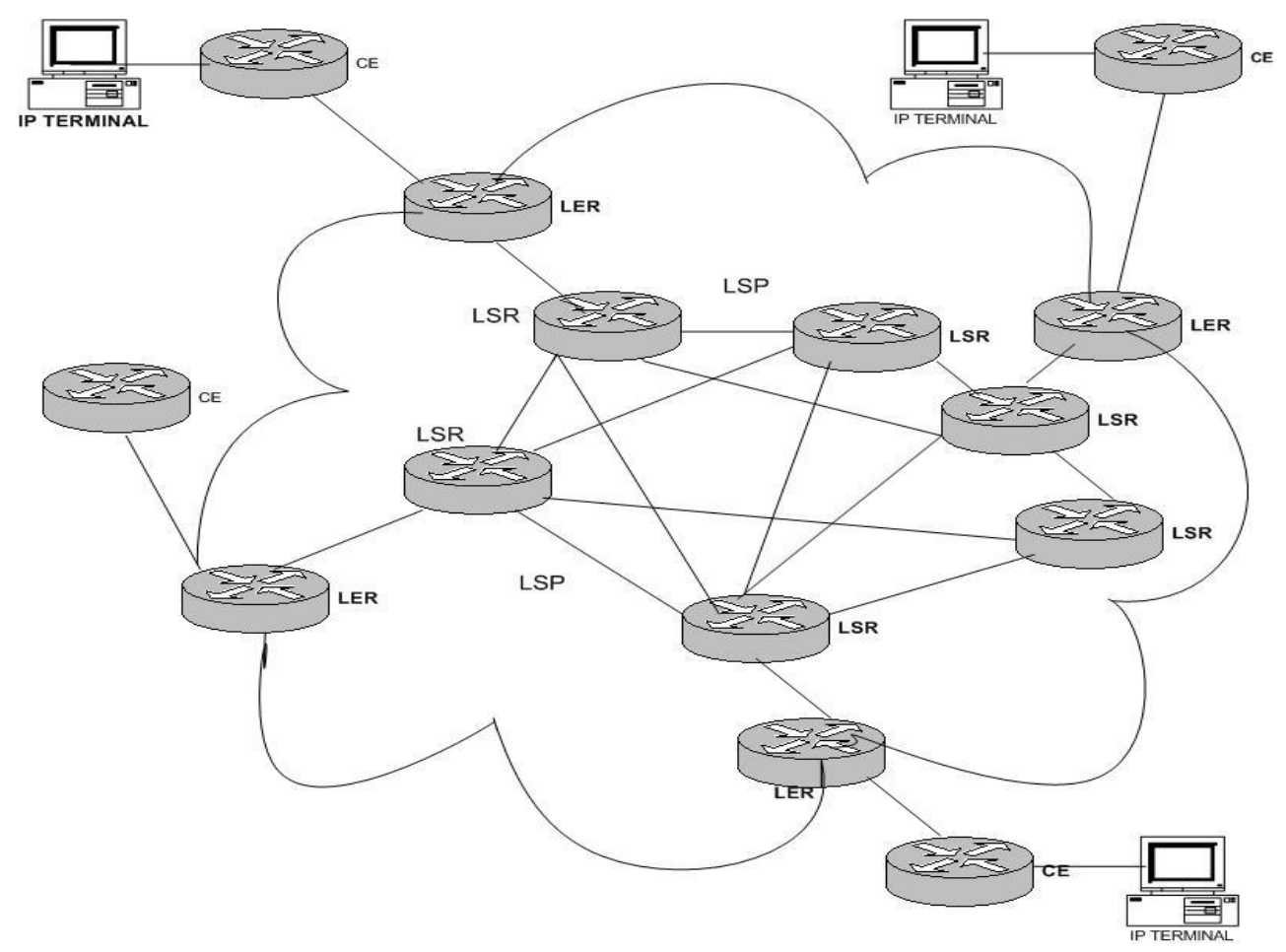

VI. CONCLUSION

Some general conclusions may be swamp from IP evolution. The change is rather inevitable. New functions of IPv6 and ICMPv6 lead to new threats. IP transition period has (and will have for many years) great impact on Internet security, performance and economy. Since all popular drill methods (Teredo, 6to4, ISATAP, tunnel broker) use IPv4 networks, the security concerns related to IPv4 are still relevant. In popular dual- stack architecture the problems resulting from IPv6 introduction may potentially have unforeseen effects on IPv4 processing, affecting both services. There are many security issues related to IPv6 deployment. perfect list of new threats and risks related to IPv6 is very long. It is probable that the list will grow longer in the future. In general the security issues related to IP transition phase may be divided into 3 classes:

- $\quad$ Related to ipv6 internal features,

- $\quad$ Related to ipv6 implementations,

- $\quad$ Related to ipv4 to ipv6 transition mechanisms.

A variety of risks and threats are results of the issues. In the previous sections we have described examples of threats from several categories:

DOS attacks, covert channels through firewalls,

- $\quad$ Privacy problems,

- $\quad$ Extra complexity of management/security tasks,

- Performance deterioration.

In the time of full IPv6 deployment IPv6 will be more than 30 years old. It is very unlikely that the protocol will be appropriate for Internet in for example, years 2020-2030.Finally, it must be said that many attacks are targeted at the appeal layer. Since the attacks are unrelated to a particular IP version IPv6 deployment will not change the security level of the application layer 
Vol. 8, Issue 3, March 2019

\section{REFERENCES}

[1]. 6net Large-Scale International IPv6 Pilot Network, 6NET Consortium, 2008, http://www.6net.org, (last access 7.03.2011).

[2]. IPv6 Fix Official Homepage, WIDE Project, 2007, http://v6fix.net/index.html, (last access 7.03.2011).

[3]. USGv6 Testing Program, NIST, 2010, http://www.antd.nist.gov/usgv6/testing.html, (last access 7.03.2011).

[4]. S. Convery and D. Miller, IPv6 and IPv4 Threat Comparison and Best Practice Evaluation, 2004, Cisco Systems, http://www.cisco.com/ security_services/ciag/documents/v6-v4- threats.pdf, (last access 30.11.2010).

[5]. E. Davies, S. Krishnan, and P. Savola, IPv6 Transition/Coexistence Security Considerations, RFC 4942, IETF, 2007.

[6]. S. Hogg and E. Vyncke, IPv6 Security, Addison Wesley, 2008.

[7]. M.P. Gallaher and B. Rowe, IPv6 Economic Impact Assessment, NIST, October, 2005.

[8]. Advancing the Internet. Action Plan for the deployment of Internet Protocol version 6 (IPv6) in Europe, Commission of the European Communities, Brussels, 2008, http://www.ipv6.eu/admin/bildbank/uploads/Documents/Commision/ COM_.pdf, (last access 30.11.2010)

[9]. M. Botterman, Towards IPv6 Deployment, RIPE 59 Lisbon, 2009,http://ripe59.ripe.net/presentations/botterman-towards-v6deployment.pdf, (last access 7.03.2011).

[10]. E. Nordmark, Basic Transition Mechanisms for IPv6 Hosts and Routers, RFC 4213, IETF, 2005. 